\title{
IX. On the criterion for the oscillatory discharge of a condenser
}

\author{
E. H. Barton D.Sc. F.R.S.E. \& W. B. Morton M.A.
}

To cite this article: E. H. Barton D.Sc. F.R.S.E. \& W. B. Morton M.A. (1899) IX. On the criterion for the oscillatory discharge of a condenser, Philosophical Magazine Series 5, 48:290, 143-147, DOI: $10.1080 / 14786449908621311$

To link to this article: http://dx.doi.org/10.1080/14786449908621311

Published online: 08 May 2009.

Submit your article to this journal $₫$

Џll Article views: 2

Q View related articles $\square$ 
Criterion for the Oscillatory Discharge of a Condenser, 143

$$
\mathrm{PQ}_{1}=\mathrm{M}_{1} \frac{x_{2}}{x_{1}}=\mathrm{RQ}_{2}=\mathrm{M}_{2}+c ;
$$

while $O Q_{2}=\mathrm{L}_{2}$ and $O \mathrm{Q}_{1}=\mathrm{L}_{1}$ are in the ratio of $x_{2}: x_{1}$.

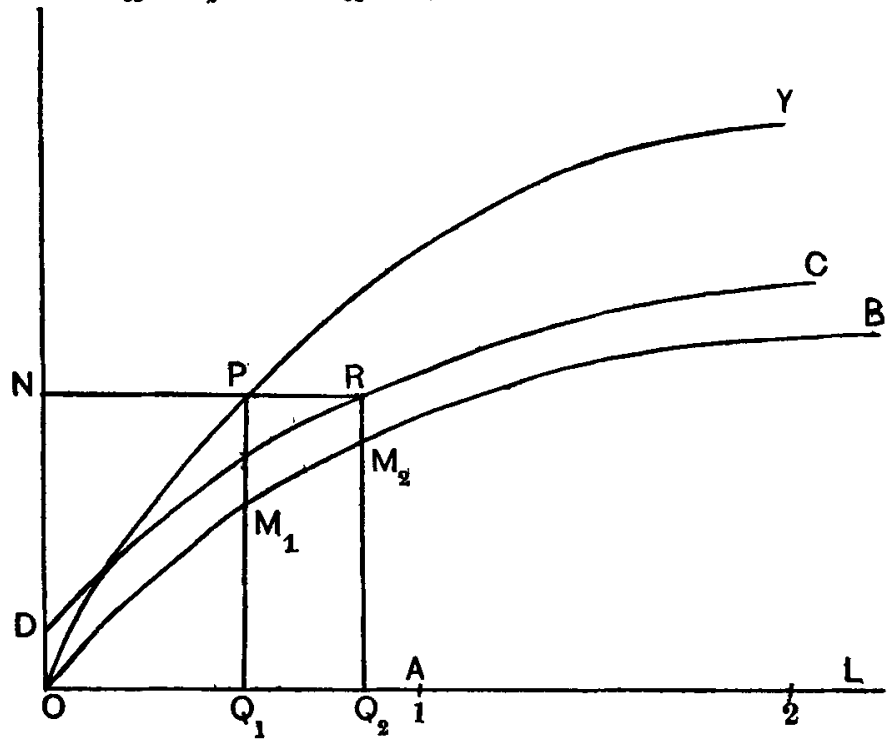

Then on the scale used $O Q_{1}$ would give a numerical value for $\frac{x_{1}}{\sqrt{4 \kappa} t}$, and $O Q_{2}$ for $\frac{x_{2}}{\sqrt{4 \kappa t}}$, from either of which $t$ might be found.

IX. On the Criterion for the Oscillatory Discharge of a Condenser. By E. H. BARTON, D.Sc., F.R.S.E., and W. B. MoRTon, M.A.*

THE ordinary condition for the oscillatory discharge of a 1 condenser of capacity $\mathrm{C}$ through a wire of resistance $\mathbf{R}$ and inductance $\mathrm{L}$, namely

$$
C<\frac{4 \mathrm{~L}}{\mathrm{R}^{2}},
$$

is obtained from the differential equation

$$
0=\frac{\mathrm{Q}}{\mathrm{C}}+\mathrm{R} \frac{d \mathrm{Q}}{d t}+\mathrm{L} \frac{d^{2} \mathrm{Q}}{d t^{2}} \text {. . . . . }
$$

by making $Q$ proportional to $e^{x t}$ and expressing the condition

* Communicated by the Physical Society : read March 24, 1899. 


\section{Dr. Barton and Mr. Morton on the Criterion jor}

that the resulting quadratic has imaginary roots. Now if account be taken of the distribution of the current in the wire, the well-known work of Maxwell and Lord Rayleigh* shows that the above differential equation must, for a straight wire, be supplemented by terms on the right,

$$
-\frac{1}{12} \mathrm{R} \alpha^{2} \mu^{2} \frac{d^{3} \mathrm{Q}}{d t^{3}}+{ }_{48}^{1} \mathrm{R} \alpha^{3} \mu^{3} \frac{d^{4} \mathrm{Q}}{d t^{4}}-{ }_{180}^{1} \mathrm{R} \alpha^{4} \mu^{4} \frac{d^{5} \mathrm{Q}}{d t^{5}}, \& c ., .
$$

where $\alpha$ is the length of the wire divided by its resistance $\left(\frac{l}{\mathrm{R}}\right)$, and $\mu$ the permeability of the material of the wire. For a curved wire we should probably have an equation of the same form with different coefficients. The question is, how do these additional terms affect the condition for oscillatory discharge?

The added terms have coefficients which are in general small compared with R. Lord Rayleigh (loc. cit.) takes, for iron, the value $10^{4}$ for resistivity and 300 for $\mu$. This would give for a wire of radius $a, \mu \alpha=\frac{3 \pi a^{2}}{100}$; so that the coefficient of $\frac{d^{3} Q}{d t^{3}}$ wonld be, even for thick wire, less than $R \times 10^{-5}$.

For copper the value would be still less. If, therefore, we put $e^{x t}$ for $Q$, we have an algebraic equation of which the terms above the second are of small and decreasing importance. The effect of these small terms will be (1) to introduce very large roots corresponding to very rapid oscillations; these will clearly he of small amplitude, and will not affect the main phenomena of discharge: (2) to modify the original roots of the uncorrected quadratic equation. The cases of oscillatory and non-oscillatory discharge are separated by the case of equality of these displaced roots.

It is easy to see that the effect of the added terms will be to make the critical value on the simple theory $\left(\mathrm{C}=\frac{4 \mathrm{~L}}{\mathrm{R}^{2}}\right)$ correspond actually to an oscillatory discharge. For in this case the graph of $y=\frac{1}{\mathrm{C}}+\mathrm{R} x+\mathrm{L} x^{2}$ evidently touches the axis of $x$ at a point on the negative side of the origin, viz. $x=-\frac{\mathbf{R}}{2 \mathbf{L}}$, and lies entirely above the axis. If we compound with this the graph of the additional terms,

$$
y=-\frac{1}{12} \mathrm{R} \alpha^{2} \mu^{2} x^{3} \& c \text {., }
$$

* Maxwell, 'Treatise,' vol. ii. $\$ 690$; Rayleigh, Phil. Mag. vol. xxi, p. $381(1886)$. 
which, for negative $x$, also lies above the axis, we get the parabolic graph displaced upwards, so that the roots of the moditied equation become imaginary.

To find to any desired degree of approximation the condition for equality of the chief roots of the equation, we may use the principle that a repeated root of an equation $f(x)=0$ is a root also of the derived equation $f^{\prime}(x)=0$.

In what follows we shall write the differential equation of discharge in the form

$$
0=\frac{\mathrm{Q}}{\mathrm{C}}+\mathrm{R} \frac{d \mathrm{Q}}{d t}+\mathrm{L} \frac{d^{2} \mathrm{Q}}{d t^{2}}+\phi\left(\frac{d}{d t}\right) \mathrm{Q}, \ldots .
$$

and the corresponding algebraic equation

$$
0=\frac{1}{\mathrm{C}}+\mathrm{R} x+\mathrm{L} x^{2}+\phi(x), \quad . \quad . \quad .
$$

using $\phi$ for the series of small terms in $\alpha^{2} \mu^{2}$, \&c.

The derived equation is

$$
0=\mathrm{R}+2 \mathrm{I} x+\phi^{\prime}(x) . \quad \cdot \quad \cdot \quad \text {. . }
$$

Let the common root of these equations be $x=-\frac{\mathrm{R}}{2 \mathrm{~L}}+\theta$, where $\theta$ is small; then we can find $\theta$ to any degree of approximation from (5), which becomes

$$
\begin{aligned}
0 & =2 \mathrm{~L} \theta+\phi^{\prime}\left(-\frac{\mathrm{R}}{2 \mathrm{~L}}+\theta\right) \\
& =2 \mathrm{~L} \theta+\phi_{0}{ }^{\prime}+\phi_{0}{ }^{\prime \prime} \cdot \theta+\frac{1}{2} \phi_{0}{ }^{\prime \prime \prime} \cdot \theta^{2}+\& c ., . .
\end{aligned}
$$

where $\phi_{0}$ is put for $\phi\left(-\frac{\mathrm{R}}{2 \mathrm{~L}}\right)$.

This gives

$$
\theta=-\frac{\phi_{0}{ }^{\prime}}{2 \mathrm{~L}}+\frac{\phi_{0}{ }^{\prime} \phi_{0}{ }^{\prime \prime}}{4 \mathrm{~L}^{2}}-\frac{\phi_{0}{ }^{\prime}}{16 \mathrm{~L}^{3}}\left(2 \phi_{0}{ }^{\prime \prime 2}+\phi_{0}{ }^{\prime} \phi_{0}{ }^{\prime \prime \prime}\right) .
$$

$\mathrm{E}_{\mathrm{l}}$ uation (4) becomes, in terms of $\theta$,

$$
0=\frac{1}{\mathrm{C}}-\frac{\mathrm{R}^{2}}{4 \mathrm{~L}}+\mathrm{L} \theta^{2}+\phi\left(-\frac{\mathrm{R}}{2 \mathrm{~L}}+\theta\right) \ldots .
$$

Putting in this the value (7), we get the required condition in the form

$$
\frac{1}{\mathrm{C}}=\frac{\mathrm{R}^{2}}{4 \mathrm{~L}}-\phi_{0}+\frac{\phi_{0}{ }^{2}}{4 \mathrm{~L}}-\frac{\phi_{0}{ }^{2} \phi_{0}{ }^{\prime \prime}}{8 \mathrm{~L}^{2}}, \ldots . .
$$

which goes to the third order in $\phi_{0}$ or the sixth in $\alpha \mu$.

Phil. Mag. S. 5. Vol. 48. No. 290. July 1899. I 
Inserting the numerical values and arranging in powers of $\alpha \mu$, it comes out

$$
\frac{1}{\mathrm{C}}=\frac{\mathrm{R}^{2}}{4 \mathrm{~L}}-\frac{1}{96} \frac{\mathrm{R}^{4} x^{2} \mu^{2}}{\mathrm{~L}^{3}}-\frac{1}{768} \frac{\mathrm{R}^{5} \alpha^{3} \mu^{3}}{\mathrm{~L}^{4}}+\frac{37}{46080} \frac{\mathrm{R}^{6} \alpha^{4} \mu^{4}}{\mathrm{~L}^{5}} .
$$

Since $\alpha=\frac{l}{\mathbf{R}}$, where $l$ is the length of the wire, we have the correcting terms expressed as a series in $\frac{\mu l}{\mathrm{~L}}$ thus

$$
\frac{1}{\mathrm{C}}=\frac{\mathrm{R}^{2}}{4 \mathrm{~L}}\left\{1-\frac{1}{24}\left(\frac{\mu l}{\mathrm{~L}}\right)^{2}-\frac{1}{192}\left(\frac{\mu l}{\mathrm{~L}}\right)^{3}+\frac{37}{11520}\left(\frac{\mu l}{\mathrm{~L}}\right)^{4} \ldots\right\} \text {. }
$$

Equations (10), (11) show that the critical capacity is greater than that given by the simple theory.

It is interesting to compare with the above another and more physical way of treating the question. In a paper by one of us * read before the Physical Society on January 27th of this year, expressions were obtained for the equivalent resistance and inductance of a wire for damped simple harmonic oscillations. The method of that paper was equivalent to putting for $Q$ in equation (3) the value $e^{(i p-k p) t}$ and arranging the result in the form

$$
0=\frac{1}{\mathrm{C}}+(i p-k p) \mathrm{R}^{\prime \prime}+(i p-k p)^{2} \mathrm{~L}^{\prime \prime} . \quad \cdot
$$

The real quantities $R^{\prime \prime}$, $L^{\prime \prime}$ gave the resistance and inductance required. To apply this to the present problem,--we can evidently get an approximation to the criterion sought by replacing $R$ and $L$ in the ordinary formula $C=\frac{4 L}{R^{2}}$ by modified values appropriate to the case. These may be got approximately by putting, in the expressions for $\mathrm{R}^{\prime \prime}$ and $\mathrm{L}^{\prime \prime}$, $p=0$ and $k p=\frac{\breve{\mathrm{R}}}{2 \mathrm{l}}$.

The result obtained by this method agrees with (11) as far as the terms there given. Let us now examine what the process sketched above really amounts to as a mathematical treatment of equation (4), and to what order of small quantities its approximation holds good.

First we must put ip $-k p$ in $\phi$ and arrange in accordance with (12). Using Taylor's expansion, we have

$$
\begin{gathered}
\phi(i p-k p)=(i p-k p) \cdot \frac{\phi(i p-k p)}{i p-k p} \\
=(i p-k p)\left[\frac{\phi(-k p)}{-k p}+i p \cdot\left\{\frac{\phi^{\prime}(-k p)}{-k p}-\frac{\phi(-k p)}{k^{2} p^{2}}\right\}+\ldots\right]
\end{gathered}
$$

* Barton, Phil. Mag. vol. xlvii. pp. 433-441 (1899). 


$$
\begin{aligned}
& =\left(i p-k_{p}\right)\left[-\frac{2 \phi(-k p)}{k p}-\phi^{\prime}(-k p)+\& c .\right] \\
& +(i p-k p)^{2}\left[-\frac{\phi(-k p)}{k^{2} p^{2}}-\frac{\phi^{\prime}(-k p)}{k p}+\& c .\right] ;
\end{aligned}
$$

all terms in the square brackets, with the exception of thoso written, vanish with $p$.

Now put $p=0, k p=\frac{\mathbf{R}}{2 \mathrm{~L}}$, and referring to the complete equation (4) we see that the "equivalent" $R$ " and $L$ " become respectively

$$
\begin{aligned}
& \mathrm{R}_{0}{ }^{\prime \prime}=\mathrm{R}-\frac{4 \mathrm{~L}}{\mathrm{R}} \phi_{0}-\phi_{0}{ }^{\prime}, \\
& \mathrm{L}_{0}{ }^{\prime \prime}=\mathrm{L}-\frac{4 \mathrm{~L}^{2}}{\mathrm{R}^{2}} \phi_{0}-\frac{2 \mathrm{~L}}{\mathrm{R}} \phi_{0}{ }^{\prime} .
\end{aligned}
$$

It may be easily verified that these expressions agree with those got from equations (15) and (16) of the paper quoted, when the values zero and $\frac{\mathrm{R}}{2 \mathrm{~L}}$ are put for $p$ and $k p$ respectively.

We see now that the use of the condition

$$
\mathrm{C}=\frac{4 \mathrm{~L}_{0}{ }^{\prime \prime}}{\mathrm{R}_{0}{ }^{\prime \prime 2}}
$$

is equivalent, mathematically, to writing equation (4) in the form

$0=\frac{1}{\mathrm{U}}+x\left\{\mathrm{R}+\frac{2 \phi(x)}{x}-\phi^{\prime}(x)\right\}+x^{2}\left\{\mathrm{~L}-\frac{\phi(x)}{x^{2}}+\frac{\phi^{\prime}(x)}{x}\right\}$, putting for $x$ inside the brackets the value $-\frac{\mathrm{L}}{2 \mathrm{R}}$, and treating the equation as a quadratic with constant coefficients.

As regards the degree of approximation given by this method, if we expand $\frac{\mathrm{R}_{0}{ }^{\prime \prime 2}}{4 \mathrm{~L}_{0}{ }^{\prime \prime}}$, we get

$$
\frac{1}{\mathrm{C}}=\frac{\mathrm{R}^{2}}{4 \mathrm{~L}}-\phi_{0}+\frac{\phi_{0}{ }^{2}}{4 \mathrm{~L}}+\frac{\phi_{0}{ }^{2}}{2 \mathrm{R}^{2}}\left(2 \mathrm{~L} \phi_{0}+\mathrm{R} \phi_{0}{ }^{\prime}\right) \ldots,
$$

which agrees with the accurate expansion in (9) up to and including terms of the second order in $\phi$. Or expressing the condition in a series of powers of $\alpha \mu$, as in (10), the two series coincide up to and including terms in $\alpha^{5} \mu^{5}$.

Nareh ?nd, 1899. 